\title{
An Investigation Capability Model for Bidirectional Pedestrian Flow
}

\author{
Mohammed Mahmod Shuaib ${ }^{1} \&$ Zarita Zainuddin ${ }^{2}$ \\ ${ }^{1}$ College of Shari'a and Islamic Studies in Al Ahsaa, Department of Computer Sciences, Al-Imam Muhammad \\ Ibn Saud Islamic University (IMSIU), Saudi Arabia \\ ${ }^{2}$ School of Mathematical Sciences, Universiti Sains Malaysia, Pulau Pinang, Malaysia \\ Correspondence: Mohammed Mahmod Shuaib, College of Shari'a and Islamic Studies in Al Ahsaa, Department \\ of Computer Sciences, Al-Imam Muhammad Ibn Saud Islamic University (IMSIU), Al Ahsaa 31982, Saudi \\ Arabia.E-mail: mh_shuaib@yahoo.com
}

Received: June 25, 2015

Accepted: July 8, 2015

Online Published: October 31, 2015

doi:10.5539/mas.v9n12p88

URL: http://dx.doi.org/10.5539/mas.v9n12p88

\begin{abstract}
The pedestrian traffic flow in bidirectional walkways is very crucial aspect influenced by the level of pedestrians' decisions. In this article, the authors show that the simulated pedestrians walking based on crowd dynamics models of low level mechanism of navigation (operational level) are short-sighted in avoiding counter flow. Such limitation resulted in unrealistic formation of motion in bidirectional flow, that the movement is less systematic and the lanes are less coherent than what in real situation. To obtain a more representative model, the authors improve the investigation capability model as a tactical decision model to be incorporated into a crowd dynamics model to reproduce better formation of motion. This is accomplished by granting the pedestrians the ability to investigate the macroscopic behaviors in their investigation areas and make decisions for convenience flow. The new model considers the average density and flow inside such areas and models their effect on the pedestrians' decisions. Simulations are performed to validate the work qualitatively by tracing the behavior of the simulated pedestrians and studying the impact of this behavior on the self-organized phenomenon: lane formation. Furthermore, the fundamental diagram of bidirectional flow is reproduced and compared with experimental fundamental diagrams.
\end{abstract}

Keywords: bidirectional flow, investigation capability, lane formation, social force model

\section{Introduction}

Over the last few decades, researchers have devoted much attention to pedestrian dynamics studies to provide solutions for some challenging problems such as massive congestion. Understanding the pedestrians walking behavior in order to eliminate the undesirable ones is a significant solution proposed to this environmental problem. A variety of crowd dynamics models has been proposed in pedestrian dynamics studies in order to represent the realistic pedestrians walking behavior, among which are the Cellular Automata Models (Blue and Adler, 2000 and Burstedde et al., 2001), Discrete Choice Models ( Antonini et al., 2006; and Robin et al., 2009), and the Social Force Model (Helbing \& Monlar, 1995; Helbing, Farkas, \& Vicsek, 2000 and Lakoba et al., 2005). A variety of methods were adopted for the purpose of validation of such crowd dynamics models, such as the introduction of the self-organization phenomena (e.g. lane-formation, arch-liked clogging at exits, and oscillations at a bottleneck in pedestrian counter flow) and the reproduction of experimental and empirical data such as the specific flow rate and the fundamental diagrams.

Bidirectional pedestrian flow is an interesting subject which has provoked the researchers to conduct a series of experimental studies to explore its aspects (Lam \& Lee, 2002; Daamen \& Hoogendoorn, 2005; Zhang, et al., 2012; and Gue, 2012). Among these aspects is lane formation (which results mainly from the natural behaviors (maneuvers) of some pedestrians when their motion is hindered by other pedestrians walking with low speed or opposite direction. Consequently, they deviate from their path to the less dense side. After deviation, the pedestrian may face the back of another pedestrian with acceptable velocity. In this case, he/she keeps walking behind the other pedestrian without any deviation. Behaving in such a way by many pedestrians will cause strip lanes to emerge automatically). Corresponding to these experimental studies, studying the pedestrians' behavior to reproduce the aspects of bidirectional flow has received much attention (Blue and Adler, 2000; Burstedde et al., 2001; Heliövaaraa et al., 2012 \& Zhang, 2015). According to Hoogendoorn, Bovy and Daamen (2001), the 
pedestrian's behavior can theoretically be divided into three inter-related levels: first, the strategic level, where the pedestrian's activities and their order are determined; second, the tactical level, where decisions are made while performing the activities (e.g., choosing a route to an intermediate target among alternative routes); and third, the operational level, where the instantaneous behaviors that involve most activities resulting from the interactions among pedestrians, such as avoiding collisions, deviations, acceleration and deceleration, are described. All aspects of this last level are examples of the pedestrian dynamics based on a variety of the aforementioned crowd dynamics models. The definition of the intermediate destination, which was exogenous input in most related studies, is the only tactical level aspect belonging to these Models. A detailed literature review of the tactical models was introduced by Daamen (2004). Hoogendoorn and Bovy (2004), Daamen (2004), Papadimitriou, Yannis and Golias (2009), and Asano, Iryo and Kuwahara (2010) pointed out the importance of obtaining integrated models comprised of two complementary levels: the operational and the tactical levels. Moreover, they demonstrated theoretically how the change in the behaviors in one level would influence the behaviors in the other. Accordingly, incorporating a route/exit choice model to grant the pedestrians of the existing microscopic model far-sighted decisions is an essential factor for obtaining a more realistic model.

Based on this view, a decision making capability for unidirectional flow has been incorporated in the Social Force Model in order to enhance the pedestrians' abilities of avoiding some anticipated blocked situations in their front areas (Zainuddin \& Shuaib, 2010). However, such capability has not been considered for bidirectional flow. In this article, we improve this investigation capability to be incorporated into the Social force Model to introduce realistic motion in bidirectional flow.

This paper is organized as follows. In the next section we introduce the decision making capability in the Social Force Model for unidirectional flow, and the arguments for the shortcomings of the model in modeling decision capability in bidirectional flow. In the third section, a model of the effects of the repulsive attributes of the pedestrian's investigation area on his/her decision is introduced. Finally, the relevant simulations to demonstrate the results of our work are performed.

\section{The Social Force Model and Decision Making Capabilities}

\subsection{The Social Force Model (SFM)}

The Social Force Model (denoted here by the SFM model) was developed by Helbing and Molnar (1995). Its most important features are its representation of pedestrian's motivations in terms of the other objects (pedestrians and obstacles) surrounding him/her as social forces. The sum of these forces is applied in a Newtonian Equation to determine the acceleration of the pedestrian's motion. An extension by Helbing et al. (2000) incorporated the physical forces arising in the case of contact amongst the pedestrians into the model. The main equations of the model are

$$
\begin{gathered}
\frac{d \vec{x}_{i}(t)}{d t}=\vec{v}_{i}(t), \\
m_{i} \frac{d \vec{v}_{i}}{d t}=\vec{f}_{i}+\varepsilon_{i}=\vec{f}_{i}^{\text {pref }}+\sum_{j} \vec{f}_{i j}+\sum_{\text {object }} \vec{f}_{i, o b j e c t}+\varepsilon_{i}, \\
\vec{f}_{i}^{\text {pref }}(t)=\gamma_{i}\left(\vec{v}_{i}^{0}(t)-\vec{v}_{i}(t)\right), \\
\vec{f}_{i j}(t)=\vec{f}_{i j}^{\text {att }}(t)+\vec{f}_{i j}^{\text {rep }}(t)+\vec{f}_{i j}^{\text {push }}(t)+\vec{f}_{i j}^{\text {friction }}(t), \\
\vec{f}_{i, o b j e c t}(t)=\vec{f}_{i, \text { abject }}^{\text {att }}(t)+\vec{f}_{i, o b j e c t}^{\text {rep }}(t)+\vec{f}_{i, \text { object }}^{\text {push }}(t)+\vec{f}_{i, \text { object }}^{\text {friction }}(t),
\end{gathered}
$$

where $\frac{d \vec{x}_{i}(t)}{d t}$ is the rate of change in the location of pedestrian $i$ at time $t ; \vec{v}_{i}(t)$ represents his/her actual velocity, which is computed numerically by solving Eq. $(2) ; \varepsilon_{i}(t)$ is a fluctuation term; the function $\vec{f}_{i}^{\text {pref }}(t)$ is the preferred force which expresses the motivation of the pedestrian $i$ to adjust his/her actual velocity $\vec{v}_{i}(t)$ to 
reach his/her preferred velocity $\vec{v}_{i}^{0}(t)=v_{i}(t) \cdot \vec{e}^{0}$; the parameter $\gamma_{i}$ is expressed as the mass of the pedestrian $i$

divided by his/her relaxation time $\tau_{i}$. In addition, the function $\vec{f}_{i j}(t)$ is the sum of all forces exerted by pedestrian $j$ upon pedestrian $i$, which are of two types. The first type is the social forces: the repulsive social force $\vec{f}_{i j}^{r e p}(t)$ and the attractive social force $\vec{f}_{i j}^{a t t}(t)$ that represent the model of the repulsive and attractive motivations inside pedestrian $i$ against and towards $j$, respectively (Helbing \& Molnar, 1995). The second type is the physical forces ( $\vec{f}_{i j}^{\text {push }}$ and $\vec{f}_{i j}^{\text {friction }}$ ), which were modeled as linear functions (Helbing et al., 2000) in analogy with the granular forces; $\vec{f}_{i, o b j e c t}(t)$ is analogous with $\vec{f}_{i j}(t)$ but with regarding objects such as walls and columns.

The authors agreed that the social forces and the calibration of its parameters are crucial for the reproduction of the relevant real-life data (Lakoba et al., 2005; Johansson et al., 2007; Alia et al., 2013; Zainuddin \& Shuaib, 2010 and Shuaib, 2014). The demonstration of the functionality of the repulsive social force is as follows. The model of the repulsive social force is based on the assumption that the pedestrian is motivated to avoid potential collisions with the surrounding objects located within his/her perception. This repulsive motivation increases with the nearness of these objects to the pedestrian's location. In the original SFM (Helbing \& Molnar, 1995), the repulsive motivation model was proposed as

$$
\vec{f}_{i j}^{r e p}(t)=A^{r e p} e^{\left(R_{i j}-d_{i j}(t)\right) / B^{r e p}} \vec{n}_{i j}(t) \cdot W\left(\phi_{i j}(t)\right),
$$

where the parameter $A^{\text {rep }}$ represents the strength of the force; the parameter $B^{\text {rep }}$ represents the repulsive distance range; $R_{i j}$ denotes the sum of the radii of $i$ and $j$ and $d_{i j}$ is the distance between the centers of $i$ and $j$; and $\vec{n}_{i j}$ is a unit vector points from the object $j$ to the pedestrian $i$. The factor $W$ is presented as a function to consider the effect the variant pedestrian's perception of the surrounding objects. It is proposed by:

$$
W\left(\phi_{i j}(t)\right)=\left(\lambda_{i}+\left(1-\lambda_{i}\right) \frac{1+\cos \left(\phi_{i j}(t)\right)}{2}\right),
$$

where $\phi_{i j}(t)$ represents the angle between the vector of pedestrian's motion $\vec{e}_{i}(t)=\vec{v}_{i}(t) /\left\|\vec{v}_{i}(t)\right\|$ and the vector $-\vec{n}_{i j}$, (i.e. $\left.\cos \phi_{i j}(t)=-\vec{n}_{i j} \cdot \vec{e}_{i}\right)$; the angular parameter $\lambda_{i}$ is to model the different effects of the pedestrian's perception to those located behind or aside him/her. Such different effects specify the shape of the anisotropic force field in the pedestrian's perception area.

In this article, we assumed the parameters $B^{r e p}$ and $\lambda$ as functions of local densities $B^{\text {rep }}(\rho)$ and $\lambda(\rho)$ as proposed by Shuaib (2014) due to the mechanisms adopted in producing these values.

\subsection{The Investigation Capability in the SFM}

The SFM has been suffering from producing counterintuitive behaviors. The simulated pedestrians in the basic SFM were too short-sighted (has limited perception), and accordingly, they cannot take a decision to avoid blocked situations resulting from several reasons such as the presence of obstacles, bottlenecks, grouped pedestrians, counter flow...ets. Generally speaking, this behavior would normally emerge in simulations based on models limited to operational decisions only. In (Smith et al., 2009) and (Pelechano et al., 2007), new terms have been incorporated into the SFM for enhancing the capability of collision avoidance. However, the pedestrian's ability is limited with avoiding the immediately adjacent object or pedestrian encountered. In simulations for unidirectional flow, Zainuddin and Shuaib (2010) granted the pedestrians the ability of avoiding the anticipated semi-blocked situations by making decisions to change directions based on their investigation of their in front areas. The investigation capability (ZS model) is represented by the feature of understanding the functionality of the density factor by the pedestrians in determining their intermediate points for their walking, accordingly changing their routes, and consequently overtaking the encountered blocked situations. The model of 
this capability, as introduced in (Zainuddin \& Shuaib, 2010), includes three essential parts:

Firstly, the investigation area model in which the pedestrian is provided with more intelligence and more options. This area is located beyond the perception area of the concerned pedestrian (see Figure 1) and is divided into parts (in front, left, and right parts) corresponding to his/her intuitive investigation which is performed by glances to the left and right of his/her original route, looking for an attractive area (lowly dense crowded area). Each part is associated with the possible route of pedestrian $i$ (left (1), forward (2), and right(3) routes) to reach his/her destination.

Secondly, the investigation process model which is based on the assumption that the pedestrian $i$ possesses his/her own awareness of the macroscopic behavior of the other pedestrians inside his/her investigation area, which may influence his/her choice of the route toward his/her destination. In other words, the pedestrian explores the repulsive characteristics of the parts (which are inherited to the included routes) of his/her investigation area, such as the density of the pedestrian crowd inside each part.

Finally, the decision making model by which the pedestrian might make a decision to direct his/her motion toward a new area which contains the route that has the minimum repulsion.

The (ZS) model has been validated by showing the lane formation phenomenon formed in the simulated unidirectional walkway (Zainuddin \& Shuaib, 2010), and by showing the intuitive realistic behaviors of the simulated pedestrians while interacting with groups of other pedestrians (Shuaib et al., 2013).

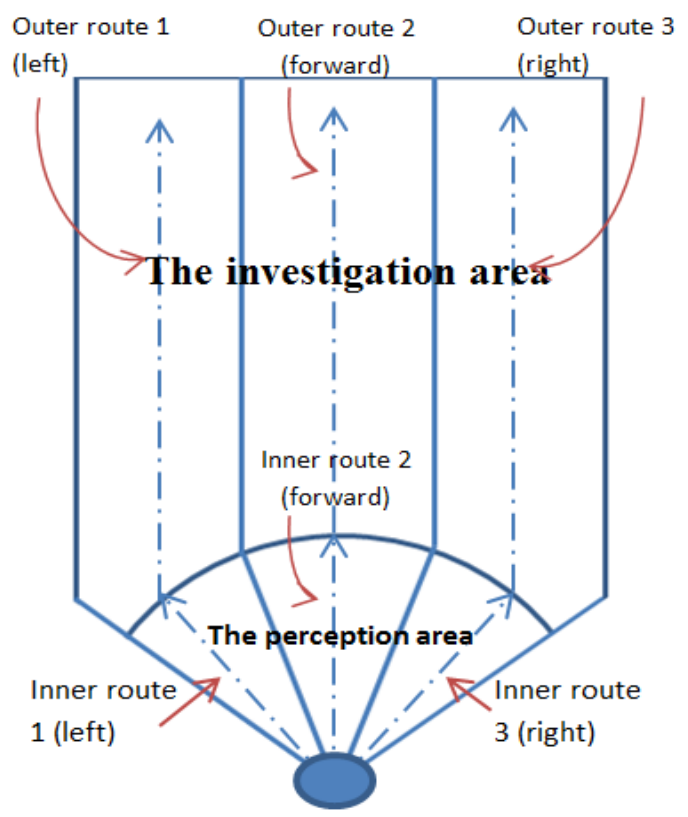

The simulated pedestrian

Figure 1. The investigation area and the route choice set. The perception area of the pedestrian is included in the investigation area, and the line is directed toward the destination. The routes are divided into two parts joined by the intermediate points chosen on the circumference of the perception area, so the model of the repulsive effect of each route is a linear combination of the repulsive effects of each part of the route

\subsection{Problematic Issue}

Concerning the implementations of the crowd dynamics models for bidirectional flow, there is still an absence of representing typical situations produced in experimental studies such as produced in (Zhang et al., 2012). The collision avoidance based on the social forces does not present the realistic aspect of lane formation as depicted in Figure 2, specially, when the pedestrians are in homogeneous bidirectional flow and not instructed to specific directions. The movement produced in the experimental study (Figure 2(b)) is more systematic and the lanes are stable and more coherent than what appears in Figure 2(a). 


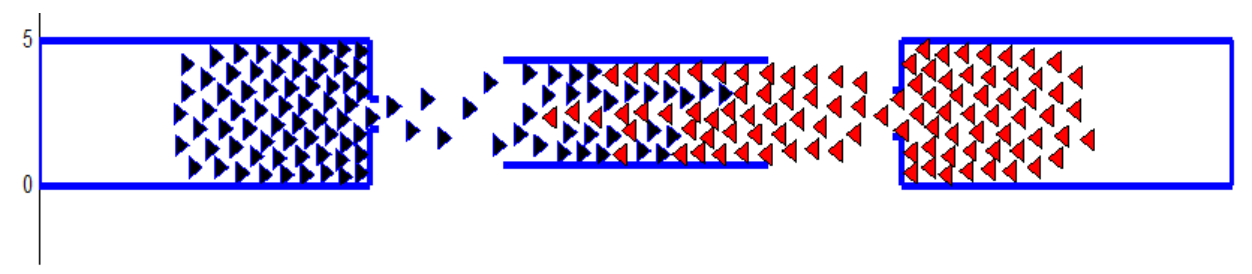

(a)
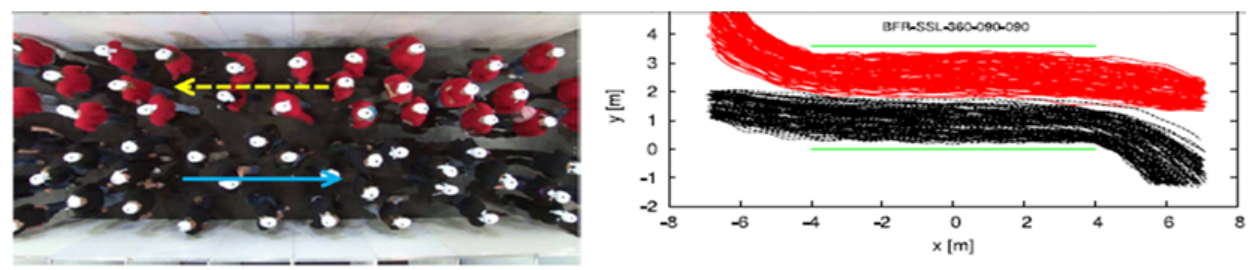

(b)

Figure 2. Snapshot (a) shows the bidirectional flow of 200 simulated pedestrians based on the SFM. The simulation was performed based on the experimental set up in (Zhang et al., 2012). In (b) bidirectional flow was produced in the experimental study (Zhang et al., 2012) based on specific ordering that the pedestrians are not instructed to particular exits. Figure 2(b) is taken from the experimental study (Zhang et al., 2012)

We believe such formation could mainly be produced by short term decisions made by the walking pedestrians in order to avoid observed counter flow within their sights. The investigation capability for bidirectional flow has not been implemented. The (ZS) model is limited with its applications (Shuaib et al., 2013) in unidirectional walkways. In the next section, we refine the (ZS) model for better representation of bidirectional flow as produced experimentally.

\section{Modeling the Investigation Process and Decision Making in a Bidirectional Walkway}

\subsection{Modeling the Excitement Factor}

The most important factor that motivates the pedestrian to look for another route (in this work, to walk in less dense crowded area or to face less counter flow) to lead him/her to his/her destination is feeling excited (uncomfortable) in his/her walking. There are many psychological factors that can influence the degree of excitement. Walking with velocity $v$ less than the preferred velocity, is an essential factor that increases the excitement factor (Helbing \& Molnar, 1995). Walking in highly crowded area beyond what the pedestrian can bear, given his/her psychological characteristics, is another factor that causes excitement. The purpose of the trip also plays a role in determining the degree of crowding with which the pedestrian will feel excited (Bovy \& Stern, 1990).

We restrict our work to the factors mentioned above and represent the model of the excitement factor $E$ for pedestrian $i$ as a linear combination of the effects of the factors $v$ and $\rho$ :

$$
E_{i}=\delta_{i}\left(v_{i}\right)+\delta_{i}\left(\rho_{i}\right),
$$

where the effects $\delta_{i}\left(v_{i}\right)$ and $\delta_{i}\left(\rho_{i}\right)$ are proposed, based on the pedestrian's walking characteristics, as follows:

$$
\begin{aligned}
& \delta_{i}\left(v_{i}\right)=\left\{\begin{array}{cc}
\frac{v_{i}-\left(v_{i}^{0}-\rho\right)}{(M-3 \rho)-\left(v_{i}^{0}-\rho\right)} & M-3 \rho \leq v_{i} \leq\left(v_{i}^{0}-\rho\right) \\
0 & \text { otherwise }
\end{array}\right. \\
& \delta_{i}\left(\rho_{i}\right)=\left\{\begin{array}{cc}
\frac{\rho_{i}-\rho_{\text {ave }} \rho_{\text {comfortable }, i}}{\rho_{\max }-\rho_{\text {ave }} \rho_{\text {comfortable }, i}} & \rho_{\text {ave }} \rho_{\text {comfortable }} \leq \rho_{i} \leq \rho_{\max } \& \quad \rho_{\text {ave }}>1 \\
0 & \text { otherwise }
\end{array},\right.
\end{aligned}
$$

where $M$ and $\sigma$ are the mean speed and the standard deviation of the Gaussian distribution produced in 
Henderson (1971) for walking speed in normal situation. The expression $v_{i}^{0}-\sigma$ is the threshold of factor $v_{i}$ such that below it places the pedestrian in an excited situation. The parameters $\rho_{\text {comfortable }}$ and $\rho_{\max }$ are found in (Syfried et al., 2005) to be $0.7 \mathrm{~m}^{-2}$ and $5.4 \mathrm{~m}^{-2}$,respectively, according to the empirical velocity-density relation of Weidmann (1991). The expression $\rho_{\text {ave }} \rho_{\text {comfortable }, i}$ is the threshold of the factor $\rho_{i}$ that exceeding it places the pedestrian in an excited situation. We reduce the effect of $\rho_{\text {comfortable }}$ by a factor $\rho_{\text {ave }}$ based on the assumption that pedestrian's pressure tolerance increases with the increase of the global density in his/her physical environment.

The pedestrian launches his investigation process when his excitement factor becomes greater than zero and he becomes motivated to investigate his/her current situation to make a decision: to keep walking on the same route or to change to another route which would (according to his evaluation) reduce his/her excitement factor.

\subsection{Modeling the Repulsion Effects of Bidirectional Flow}

Given a bidirectional walkway, we introduce the model of the flow for the pedestrian who becomes engaged with this process (i.e. $E>0$ ). His goal is to find the best route that will lead to less density pressure or less counter flow, and to direct his/her motion toward it. Accordingly, he/she is assumed to account for the repulsive effects of the available routes of his/her subjective route set.

Passing through less dense crowded areas is a goal for those who do not like highly dense crowds. For that reasons, we account for the repulsion of the routes by computing the average density of the total cells through which the route passes.

$$
\text { Density_rep } \text { route }_{j, i}(t)=d_{\text {inn }_{-} \text {route }_{j}} \cdot \rho_{\text {inn_cell }_{j, i}}(t)+d_{\text {out }_{-} \text {route }_{j}} \cdot \rho_{\text {out }_{-} \text {cell } j, i}(t),
$$

where $\rho_{\text {inn_cell }}(t)$ and $\rho_{\text {out }}$ cell $l_{j, i}(t)$ are the average densities of the inner and outer cells of the relevant route $j$, respectively; $d_{\text {inn }_{-} \text {rout }_{j}}$ and $d_{\text {out }_{-} \text {rout }_{j}}$ are the lengths of the inner and outer parts of route $j$.

On the other hand, avoiding counter flow is a main goal for those who are looking for higher velocity and less collision, we account for the repulsion of the routes by computing the counter flow within the cells through which the route passes.

$$
\text { Flow_rep } \text { route } j, i_{j}(t)=d_{\text {inn_route }_{j}} \cdot f_{\text {inn }_{-} \text {cell } j_{j, i}}(t)+d_{\text {out }_{-} \text {route }} \cdot f_{\text {out }_{-} \text {cell }_{j, i}}(t)
$$

where $f_{\text {inn_cell }_{i}}$ and $f_{\text {out }_{\text {cell }}}$ are the average counter flows inside the inner and outer cells of the relevant route $j$ at time $t$. So the model of the repulsion effect of each route is a linear combination of the different repulsions weighted by the corresponding factors of excitement

$$
\text { Repulsion }_{\text {route }_{j, i}}=\delta_{i}\left(\rho_{i}\right) \cdot \text { Density_rep } \text { route }_{j, i}+\delta_{i}\left(v_{i}\right) \cdot \text { Flow_rep }_{\text {route }_{j, i}}
$$

\subsection{Decision Making for New Preferred Direction}

The route with the minimum repulsion, which will probably be chosen by pedestrian $i$ as his/her new route $\vec{e}_{n e w, i}^{0}$ if its utility is sufficient, is determined by comparison between the computed repulsions of the subjective alternative routes and selecting the minimum repulsive route. The pedestrian makes a decision to change his/her route when the repulsion of the new route is less than the current route with worthwhile magnitude $g_{i}$, as stated by Helbing (1997) and Senevarante and Morall (1986). The preferred velocity is replaced by

$$
\begin{gathered}
\vec{v}_{i}^{0}(t)=v_{i}^{0}(t) \vec{e}_{\text {curr }, i}^{0}, \\
\vec{e}_{\text {curr }, i}^{0}(t)=\left\{\begin{array}{lc}
\vec{e}_{n e w, i}^{0} & \text { repusion } \\
\vec{e}_{\text {cur }, i}^{0}-\text { repulsion }, i & \text { otherwise }, i
\end{array} g_{i}\right.
\end{gathered} .
$$

The pedestrian continues to investigate his/her lattice, except in the case when he is walking toward his/her destination and the excitement factor is equal to zero. The situation surrounding him/her is dynamic; a movable intermediate point may disappear because of the dynamic traffic. By continuous investigation, the pedestrian will keep trace the repulsive aspects of his/her investigation area. 


\section{Simulation Results and Discussion}

\subsection{Simulations of Bidirectional Flow}

The simulations in this section were conducted to trace the behavior of the simulated pedestrians who possess the investigation ability, and to study the impact of this behavior on the self-organized phenomenon (lane formation) as introduced in (Zhang et al., 2012). Different types of bidirectional flow have been treated in (Zhang et al., 2012). However, we focus on the ordering where the pedestrians were not instructed about the exit to leave the corridor. The physical environment depicted in (Figure 3) is set up according to the experimental set up performed in (Zhang et al., 2012): straight corridor with widths of $3.6 \mathrm{~m}$. The entrance width for both directions is the same. The positions of the simulated pedestrians were initialized in waiting areas at the left and right sides of the corridor, and their preferred velocities are Gaussian distributed (Henderson, 1971), with mean equal to $1.34 \mathrm{~m} / \mathrm{s}$ and standard deviation equal to $0.26 \mathrm{~m} / \mathrm{s}$. The pedestrians' parameters, the Social Force Model parameters and the new parameters are shown in Table 1.

Table 1. The parameters' values

\begin{tabular}{lr}
\hline$m=[77-83] \mathrm{kg}$ & The range of pedestrians' mass \\
\hline$r=[0.25-0.30] \mathrm{m}$ & The range of pedestrians' radius \\
$A^{\text {rep }}=2000 \mathrm{~N}$ & The strength parameter \\
$B^{\text {rep }}=B^{\text {rep }}(\rho)$ & The repulsive distance parameter \\
$\lambda=\lambda(\rho)$ & The angular parameter \\
$\tau=0.5 \mathrm{~s}$ & The pedestrian reaction time \\
$\varepsilon \in\left[0,0.05 * v^{0} / \tau\right]$ & The range of fluctuation source \\
$g=0.2 * r e p_{\text {curr }}$ & The threshold of utility to make a decision \\
\hline
\end{tabular}

Due to the lack of relevant psychological studies, the parameter $g_{i}$ of the proposed model presented in Table 1 were estimated by performing the simulations several times and obtaining the best behavior, based on intuition and knowledge inspired by real observations.

\subsection{Discussion}

The snapshots in (Figure 3) show that the movement of pedestrians is more systematic and the lanes are more coherent than what appears in Figure 2(a). The reason for this is that the pedestrian who has an investigation ability performs long-sighted investigation process which helps detect far-distance areas and join other pedestrians with the same flow direction, consequently, join their lane. This variation in the results between the original model (SFM) and our improved (ZS) model has also been obtained in simulations with higher and smaller number of simulated pedestrians as long as they have the same ordering. The effect of density did not play a rule here because of the regular distribution of the pedestrians' locations inside the corridor. The rule of the density could obviously emerge in irregular distribution of the pedestrians' location. 


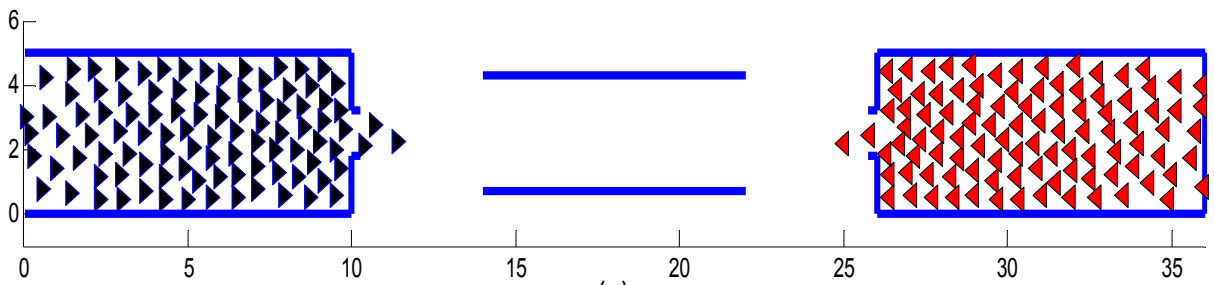

(a)

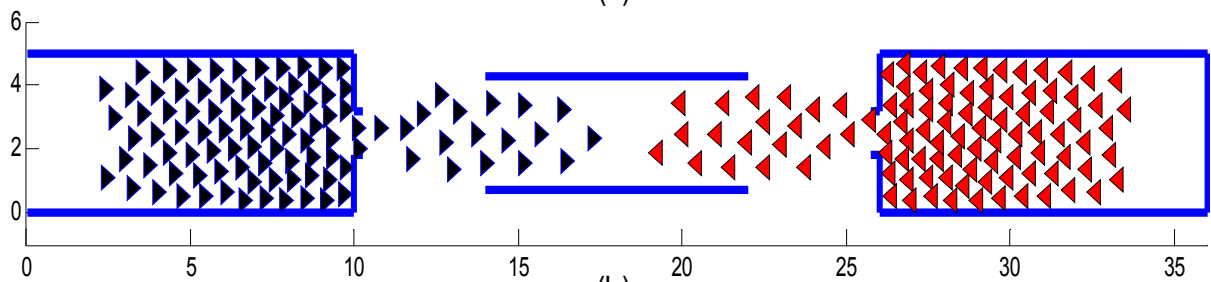

(b)

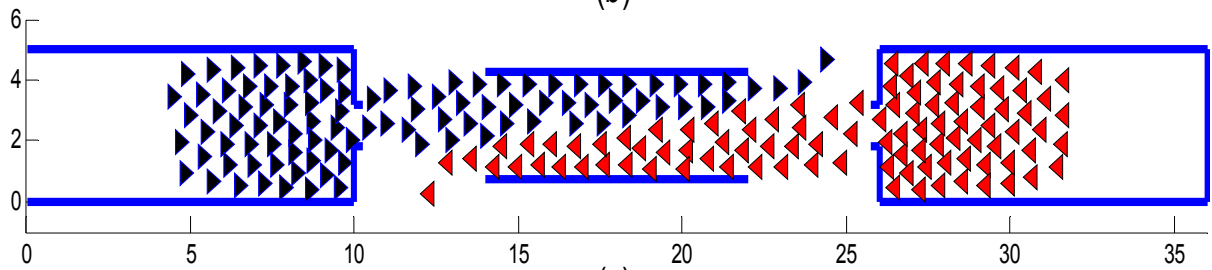

(c)

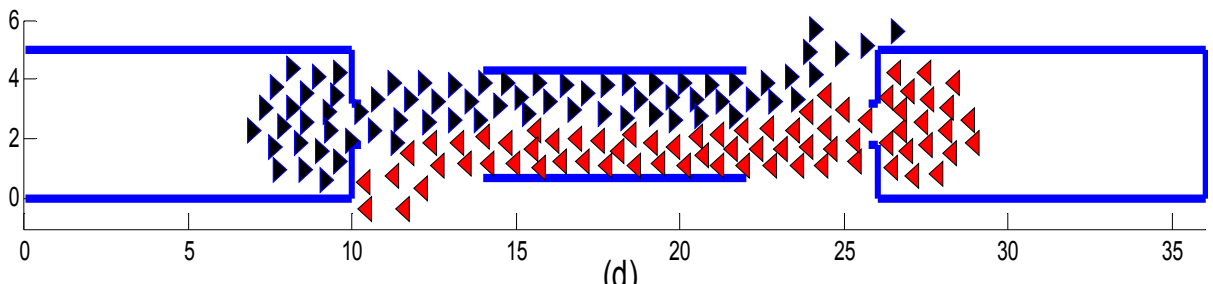

(d)

Figure 3. Four snapshots were taken from the simulations of the improved (ZS) model. Snapshots (c) and (d) show the formation of stable and separated lanes

\subsection{The fundamental Diagram}

The fundamental diagrams of unidirectional and bidirectional streams (among which are Fruin (1971), Sarkar et al. (1997), Older (1968) and Weidmann (1993)) form essential tools for the assessment of the models; whether it can describe the pedestrian stream appropriately, with respect to the empirical studies. Furthermore, it has a lot of benefits for the adjustment process of some parameters of the model in consideration (Syfried et al., 2006; Parisi et al., 2009; and Zainuddin \& Shuaib, 2010). Reproducing experimental fundamental diagrams of various ordering in bidirectional streams was done in (Zhang et al., 2012). Comparing the resulting diagrams with those of unidirectional streams, It was shown that they are slightly different, specifically, when the density value is larger than $1\left(\mathrm{~m}^{-2}\right)$.

To reproduce the fundamental diagram, we calculated the average velocities for various densities less than $3.5 \mathrm{~m}^{-2}$. As shown in Figure 4, the data (velocity-density points) was obtained and compared with the fundamental diagram of Weidmann (1993). It can be seen that the resulting fundamental diagram is in good agreement with the estimated fundamental diagram of weidmann, but with slight reduction of velocity, specifically, when the density value is larger than $1\left(\mathrm{~m}^{-2}\right)$. This result is consistent with what is stated above by Zhang et al. (2012). 


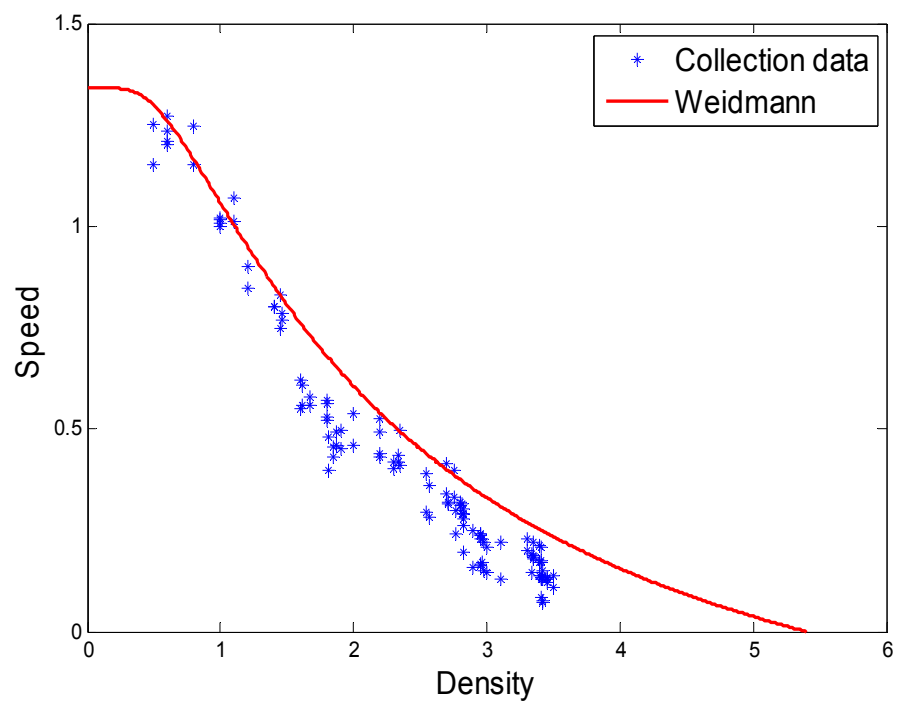

Figure 4. The velocity versus density points obtained for bidirectional flow based on the improved (ZS) model, and comparison with Weidmman relation

\section{Conclusion}

In this article, an improved tactical aspect of decision-making has been incorporated into the Social Force Model of pedestrian behavior. The pedestrians explore the area under their sights to avoid counter flow and consequently to look for more comfortable lanes to walk in. A validation of this work was introduced by showing the lane formation phenomenon, as presented in the experimental study (Zhang et al. 2012). The comparison has shown that the improved (ZS) model has two advantages: first, the flow of the pedestrians in the preferred direction is more efficient, and second, the occurrence of blocked situations is less likely to happen. These results have agreed with the qualitative validation for the aforementioned feature regarding the lane-formation phenomenon.

The work of this article can be easily extended to involve many aspects of pedestrian flow by considering different ordering of directional flow and incorporating some aspects such as a semi-blocked situation or physical characteristics such as obstacles and walls.

\section{Acknowledgements}

We thank the Al-Imam Muhammad Ibn Saud Islamic University for supporting this work.

\section{References}

Alia, O., \& Shuaib, M. M. (2014). Harmony search algorithm for the reproduction of experimental data in the social force model. J. Appl. Math. http://dx.doi.org/10.1155/2014/954607

Antonini, G., Bierlaire, M., \& Weber, M. (2006). Discrete choice models of pedestrian walking behavior. Transportation Research Part B, 40, 667-687. http://dx.doi.org/10.1016/j.trb.2005.09.006

Asano, M., Iryo, T., \& Kuwahara, M. (2010). Microscopic pedestrian simulation model combined with a tactical model for route choice behavior. Transportation Research Part c, 18, 842-855. http://dx.doi.org/10.1016/j.trc.2010.01.005

Blue, V., \& Adler, J. (2000). Cellular automata microsimulation of bi-directional pedestrian flows. Journal of the Transportation Research Board, 1678, 135-141. http://dx.doi.org/10.3141/1678-17

Bovy, P. H. L., \& Stern, E. (1990). Route Choice: Wayfinding in Transport Networks. Kluwer Academic Publishers, Dordrecht.

Burstedde, C., Klauck, K., Schadschneider, A., \& Zittartz, J. (2001). Simulation of pedestrian dynamics using a

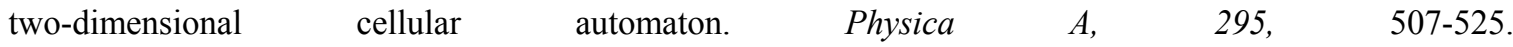
http://dx.doi.org/10.1016/S0378-4371(01)00141-8

Daamen, W. (2004). Modelling Passenger Flows in Public Transport Facilities. Ph. D. Thesis, Delft University of 
Technology, Netherlands.

Daamen, W., \& Hoogendoorn, S. P. (2005). Experimental research of pedestrian walking behavior. Transp. Res. Rec., 1828, 20-30.

Fruin, J. J. (1971a). Designing for pedestrians: A level of service concept. Highway Research Record, 355, 1-15.

Fruin, J. J. (1971b). Pedestrian planning and design. New York: Metropolitan association of urban Designers and Environmental Planners.

Guo, R. Y., Wong, S. C., Xia, Y. H., Huang, H. J., Lam, W. H. K., \& Choi, K. (2012). Empirical evidence for the look-ahead behavior of pedestrians in bi-directional flows. Chin. Phys. Lett., 29(6), 1-4. http://dx.doi.org/10.1088/0256-307X/29/6/068901

Helbing, D. (1997). Traffic Dynamics: New Physical Modelling Concepts. Springer-Verlag, Berlin.

Helbing, D., \& Molnár, P. (1995). Social Force Model for pedestrian dynamics. Physical Review E., 51, 4282-7. http://dx.doi.org/10.1103/PhysRevE.51.4282

Helbing, D., Farkas, I., \& Vicsek, T. (2000). Simulating dynamical features of escape panic. Nature, 407, 487-90. http://dx.doi.org/10.1038/35035023

Heliövaaraa, S., Korhonen, T., Hostikka, S., \& Ehtamo, H. (2012). Counterflow model for agent-based simulation of crowd dynamics, Building and Environment, 48, 89-100. http://dx.doi.org/10.1016/j.buildenv.2011.08.020

Henderson, L. F. (1971). The statistics of crowd fluids. Nature, 229, 381-383. http://dx.doi.org/10.1038/229381a0

Hoogendoorn, S P., \& Bovy, PHL. (2004). Pedestrian route-choice and activity scheduling theory and models. $\begin{array}{llll}\text { Transportation Research Part B: } & \text { Methodological, }\end{array}$ http://dx.doi.org/10.1016/S0191-2615(03)00007-9

Johansson, A., Helbing, D., \& Shukla, P. K. (2007). Specification of the social force pedestrian model by evolutionary adjustment to video tracking data. Adv Complex Syst., 10, 271-288. http://dx.doi.org/10.1142/S0219525907001355

Lakoba, T. I., Kaup, D. J., \& Finkelstein, N. M. (2005). Modifications of the Helbing-Molnar-Farkas-Vicsek Social Force Model for Pedestrian Evolution. SIMULATION, $81, \quad 339$. http://dx.doi.org/10.1016/j.physa.2009.05.027

Lam, W. H. K, \& Lee, J. Y. S. (2002). A study of the bi-directional pedestrian flow characteristics at Hong Kong signalized crosswalk facilities. Transportation, 29(2), 169-192.

Older, S. J. (1968). Movement of pedestrians on footways in shopping streets. Traffic Eng. and Control, IO, 160-163.

Papadimitriou, Y., \& Golias. (2009). A critical assessment of pedestrian behaviour models. Transportation research part F: traffic psychology and behavior, 12(3), 242-255. http://dx.doi.org/10.1016/j.trf.2008.12.004

Parisi, D., Gilman, M., \& Moldovan, H. (2009). A modification of the Social Force Model can reproduce experimental data of pedestrian flows in normal conditions. Physica A, 388, 3600-3608. http://dx.doi.org/10.1016/j.physa.2009.05.027

Pelechano, N., Allbeck, J., \& Badler, N. (2007). Controlling individual agents in high-density crowd simulation. In: ACM SIGGRAPH/Eurographics symposium on computer animation (SCA'07), p. 99e108.

Robin, T., Antonini, G., Bierlaire, M., \& Cruz, J. (2009). Specification, estimation and validation of a pedestrian walking behavior model. Transportation Research Part B: Methodological, 43(1), 36. http://dx.doi.org/10.1016/j.trb.2008.06.010

Sarkar, A. K., \& Janardhan, K. S. V. S. (1997). A study on pedestrian flow characteristics. In: CD-ROM with Proceedings. Washington: Transportation Research Board.

Sefried, A., Steffen, B., Klingsch, W., \& Boltes, M. (2005). The fundamental diagram of pedestrian movement revisited. J. Stat. Mech., P10002. http://dx.doi.org/10.1088/1742-5468/2005/10/P10002

Senevarante, P. N., \& Morall, J. F. (1986). Analysis of Factors affecting the Choice of Route of Pedestrians. Transportation Planning and Technology, 10, 147-159. 
Seyfried, A. Steffen, B., \& Lippert, T. (2006). Basics of modeling the pedestrian flow. Physica A, 368, 232_238. http://dx.doi.org/10.1016/j.physa.2005.11.052

Shuaib, M. M., Alia, O. M., \& Zainuddin, Z. (2013). Incorporating prediction factor into the investigation capability in the social force model: application on avoiding grouped pedestrians, Applied Mathematics \& Information Sciences, 7(1), 323-331.

Shuaib, M. M. (2014). Preserving Socially Expected Crowd Density in front of the Exit for the Reproduction of Experimental Data by Modeling the Pedestrians' behind Perception. J. Stat. Mech., P10037. http://dx.doi.org/10.1088/1742-5468/2014/10/P10037

Smith A., James C., Jones R., Langston P., Lester E., \& Drury J. (2009). Modelling contra-flow in crowd dynamics DEM simulation. Safety Sci., 47, 395-404. http://dx.doi.org/10.1016/j.ssci.2008.05.006

Weidmann, U., \& Transporttechnik der Fussg"anger. (1993). ETH-Z"urich, Schriftenreihe des Institut f"ur Verkehrsplanung, Transporttechnik, Strassen- und Eisenbahnbau Z"urich (in German).

Zainuddin, Z., \& Shuaib, M. (2010). Incorporating Decision Making Capability into the Social Force Model in Unidirectional Flow. Research Journal of Applied Sciences, 5(6), 388-393. http://dx.doi.org/10.3923/rjasci.2010.388.393

Zhang, G. (2012). Simulation model of bi-directional pedestrian considering potential effect ahead and behind. Physica A 419 (2015) 335-348. http://dx.doi.org/10.1016/j.physa.2014.09.054

Zhang, J., Klingsch, W., Schadschneider, A., \& Seyfried, A. (2012). Ordering in bidirectional pedestrian flows and its influence on the fundamental diagram, J. Stat. Mech., 02002. http://dx.doi.org/10.1088/1742-5468/2012/02/P02002

\section{Copyrights}

Copyright for this article is retained by the author(s), with first publication rights granted to the journal.

This is an open-access article distributed under the terms and conditions of the Creative Commons Attribution license (http://creativecommons.org/licenses/by/3.0/). 